\title{
Morfologia externa e morfometria de fetos caninos da raça pinscher aos 45 dias pós-coito
}

\section{Esternal morphology and morphometry of canine fetuses of the pinscher breed to 45 days post-coitus}

\author{
Daysianny de Oliveira Bezerra ${ }^{1}$; Heliana de Barros Fernandes ${ }^{2}$; \\ Aírton Mendes Conde Júnior ${ }^{3}$; Luana de Oliveira Lopes ${ }^{2 *}$
}

Resumo

Foram analisados aspectos morfológicos externos e morfométricos de fetos caninos da raça pinscher aos 45 dias pós-coito. Utilizaram-se quatro fetos, duas fêmeas e dois machos de uma fêmea da raça pinscher, com registro de raça, clinicamente sadia e com gestação acompanha por exames laboratoriais e sonogramas. O estudo foi realizado no laboratório de Embriologia-UFPI/CCS, após o óbito por trauma mecânico sofrido pela mãe devido a acidente automobilístico. As características morfológicas externas foram descritas e dados como peso, comprimento (total, caudal, cefálico, auricular e céfalo-caudal), crown-rump $(\mathrm{CR})$, perímetro (ocular, torácico e abdominal) e diâmetro biparietal foram obtidos. Os fetos, aos 45 dias, apresentaram pele coberta por pêlos, cabeça e membros formados e diferenciados, além de olhos fechados. Observou-se presença de pêlos tácteis, filtro labial, narinas abertas e mufla queratinizada. Possuem membros torácicos com cinco dígitos e pélvicos com quatro dígitos com unhas formadas. Apresentaram dimorfismo sexual. O peso médio dos fetos foi $56,9 \mathrm{~g} \pm 15,7$; o comprimento total $18 \mathrm{~cm} \pm 0,9 ;$ o CR $10 \mathrm{~cm} \pm 0,9$; o comprimento cefálico $4,2 \mathrm{~cm} \pm 0,2$; comprimento da cauda $3,4 \mathrm{~cm} \pm 0,4$; comprimento auricular $0,8 \mathrm{~cm} \pm 0,2$; comprimento céfalo-caudal $9,4 \mathrm{~cm} \pm 2,1$; perímetro ocular $0,5 \mathrm{~cm} \pm 0,1$; perímetro torácico $8,9 \mathrm{~cm} \pm 1,0$ e diâmetro biparietal $3,7 \mathrm{~cm} \pm 0,6$. Fetos de cães da raça pinscher têm características morfológicas e morfométricas próprias da raça e do período gestacional.

Palavras-chave: Morfologia, morfometria, fetos, pinscher

\begin{abstract}
We analyzed the external morphology and morphometric fetal pinscher dog breed at 45 days post-coitus. We used four fetuses, two females and two males from a female pinscher breed with breed registry, with clinically healthy and accompanying pregnancy by blood tests and sonograms. The study was performed at the Embriologia-UFPI/CCS after death due to mechanical trauma suffered by his mother due to car accidents. The morphological characteristics were described and data such as weight, length (total, tail, head, ear and head-tail), crown-rump (CR), perimeter (eye, chest and abdominal) and biparietal diameter were obtained. Fetuses at 45 days showed skin covered by hair, head and members formed and differentiated, and eyes closed. We observed the presence of tactile hairs, philtrum, open nostrils and muffle keratinized. They have five digits in forelimbs and hind four digits with nails formed. Showed sexual dimorphism. The average weight of fetuses was $56.9 \mathrm{~g} \pm 15.7$; the total length of $18 \mathrm{~cm} \pm 0.9$; CR $10 \mathrm{~cm} \pm 0.9$; head length $4.2 \mathrm{~cm} \pm 0.2$; tail length $3.4 \mathrm{~cm} \pm 0$, 4; ear length $0.8 \mathrm{~cm} \pm 0.2$; cephalo-caudal legth $9.4 \mathrm{~cm} \pm 2.1$; perimeter ocular $0.5 \mathrm{~cm} \pm 0.1$; thoracic perimeter $8.9 \pm 1.0$ and biparietal diameter $3.7 \mathrm{~cm} \pm 0.6$. Fetuses pinscher dogs have morphological and morphometric own race and gestational age.

Key words: Morphology, morphometry, fetuses, pinscher
\end{abstract}

1 Discente de Mestrado em Ciência Animal, Universidade Federal do Piauí, UFPI, Teresina, PI. E-mail: daysebez@hotmail.com

2 Discente(s) de Mestrado em Biotecnologia, UFPI, Parnaíba, PI. E-mail: hemtropya@yahoo.com.br; luanalopesss@hotmail.com

3 Prof. do Dept ${ }^{\circ}$ de Morfologia, Setor de Histologia e Embriologia, UFPI, Teresina, PI. E-mail: airtoncondejr@uol.com.br

Autor para correspondência 
Com uma expectativa de vida de cerca de 15 anos de idade, os cães da raça Pinscher são animais de pequeno porte. Podendo chegar, no adulto, a uma altura de 25 a $30 \mathrm{~cm}$ da cernelha com um peso médio de 4 a $6 \mathrm{Kg}$. (CONFEDERAÇÃO BRASILEIRA DE CINOFILIA, 2007).

Os pinschers possuem características bem marcantes que evidenciam a raça, não só pelo seu tamanho, mas também pelos padrões de coloração e pelagem. Os cães desta raça são largamente procurados como animais de companhia, pois possuem um temperamento bem marcante: são ousados, corajosos, curiosos e apegados e adaptam-se facilmente em ambientes pequenos como, por exemplo, apartamentos. (TEIXEIRA, 2000). Devido sua relação com o homem, os animais de companhia são os alvos preferidos das investigações científicas em medicina veterinária. Porém, pesquisas específicas às raças caninas são dificultadas pela disponibilidade de animais puros, visto o alto valor comercial que possuem.

Um dos primeiros passos para o estudo de uma espécie animal é o estudo morfológico, pois através destes dados é possível estabelecer relações que permitem a identificação da origem evolutiva deste grupo, além de dar base a estudos aplicados (KERSWELL et. al., 2009). Estudos morfológicos proporcionam conhecimentos sobre doenças relacionadas à anormalidades funcionais ou congênitas nestes animais. (FERREIRA et al, 2007; BURBIDGE; THOMPSON; HODGE, 1995).

Tão importante quanto a análise morfológica do adulto é a análise do feto e do recém nascido (DYCE; SACK; WENSING, 1997). Sabe-se que o período gestacional é uma etapa do desenvolvimento animal em crescente relevância para a medicina veterinária, pois é neste período que muitas alterações morfológicas são diagnosticadas. Em animais da raça Pinscher este período dura em torno de 65 dias (EDMONDSON, 2007). Da mesma forma, que permite estabelecer relações diretas, entre diversas doenças e o período pré-natal. (BARRETO, 2003).
A padronização de medidas morfométricas de fetos caninos viabiliza conhecimentos de normalidade morfológica necessários para guiarem métodos diagnósticos. (MOURA et. al., 2006). Para Kolber e Borelli (2005), um dos métodos diagnósticos prejudicados pela falta de padrões morfométricos de normalidade é a ultra-sonografia. Hoje, este exame é muito utilizado na clínica de pequenos animais para o acompanhamento gestacional, principalmente por ser um método não invasivo e capaz de avaliar de forma eficaz a viabilidade do concepto (CASTRO et. al., 2011).

Estudos da morfologia de fetos da raça pinscher são inexistentes, assim como de muitas raças puras no mundo. Por isso, padrões morfométricos da raça para guiar a realização de exames ultrassonográficos não estão disponíveis para os profissionais da área. Este estudo objetiva a análise morfológica e morfométrica de fetos caninos da raça Pinscher aos 45 dias pós-coito, com o intuito gerar os padrões morfológicos desta raça e permitir conhecimentos da morfologia externa e morfometria destes cães normais, bem como guiar exames ultrasonográficos e possibilitar o diagnóstico de alterações morfológicas nestes animais.

Foram utilizados quatro fetos, duas fêmeas e dois machos de uma fêmea da raça pinscher, com registro de raça, clinicamente sadia e com gestação acompanhada por exames laboratoriais e sonogramas. O estudo foi realizado no laboratório de Embriologia da Universidade Federal do Piauí Centro de Ciências da Saúde UFPI/CCS. Após óbito dos animais por trauma mecânico sofrido pela fêmea gestante devido a acidente automobilístico estes foram levados ao laboratório, pesados, identificados e foram submetidos à técnicas de morfometria para obtenção de dados morfométricos como peso em gramas, comprimento total, caudal, cefálico, auricular e céfalo-caudal, crow-rump (medido da base da cabeça à base da cauda), perímetro ocular, perímetro torácico, perímetro abdominal e diâmetro biparietal, todos em centímetros. Os dados morfométricos foram obtidos conforme sugestão de Mandarim de Lacerda (1994). 
Os animais estudados apresentaram corpo dividido em cabeça, tronco, membros torácicos e pélvicos. A cabeça apresenta formato elipsóide com dois olhos formados, ainda fortemente fechados por pálpebras. O pavilhão auricular externo e o ouvido estão formados, sendo que o ouvido está obliterado. Na região nasal existem pêlos tácteis, filtro labial, e as narinas estão abertas, com nariz queratinizado. A boca, em relação ao corpo, é relativamente grande e aberta, com língua formada. Os fetos, nesse período gestacional, não apresentam dentes. Quanto ao corpo, o membro torácico apresenta cinco dígitos e o pélvico quatro dígitos, todos com unhas formadas já aos 45 dias.

Esses dados são importantes para a definição da raça em estudo e são dificilmente encontrados na literatura, pois os dados com respeito a este período advêm de exames ultrasonográficos. Por exemplo, nos cães da raça Yorkishire Terrier pode ser observado, aos 45 dias, apenas as órbitas oculares já formadas, por ultasonografia, sem inferir a presença dos olhos e pálpebras do animal (LEITE, 2003). Além disso, Segundo Penna (2009), características morfológicas simples, como morfometria externa, e distribuição dos pêlos servem como caracterização das espécies contribuindo assim, para diferenciações taxonômicas.

Nenhuns dos cães estudados apresentaram qualquer anormalidade congênita. Anomalias que acontecem frequentemente nos filhotes, como Legg Calvé Perthes (LCP), macrocefalia e hidrocefalia podem ser detectadas pelo estudo morfológico (CASTRO et al., 2011). Como supomos que patologias desta natureza tenham origem devido a predisposições genéticas, acreditamos, assim como Stulberg, Cooperman e Wallensten (1981) que, é possível que o cão afetado já exiba sinais clínicos desde o período fetal.
Nos animais estudados, a coloração da pele era preta e castanha, como a coloração descrita no registro de raça para os pais. Esta coloração, mais uma vez, é um indicador característico da raça e os animais já apresentam este padrão de coloração ainda no período fetal. Sabe-se que quanto à coloração diferenciam-se dois tipos básicos de pinscher miniatura, o unicolor (tons vermelhocervo, de vermelho-amarronzado a vermelho marrom escuro) e preto e castanho (tons de pêlo negro com manchas marrons ou vermelhas). As manchas se localizam: sobre os olhos, na parte inferior do pescoço, nos metacarpos, nos pés, nas partes internas dos membros posteriores e no períneo. (CONFEDERAÇÃO BRASILEIRA DE CINOFILIA, 2007).

Estudamos quatro cães, dois machos e duas fêmeas sem qualquer anomalia sexual. A genitália externa está totalmente formada nos machos e nas fêmeas, podendo diferenciar-se a vulva, nas fêmeas, e o escroto e prepúcio, nos machos. Isso demonstra um estágio de avançado desenvolvimento dos órgãos sexuais, assim como explica Romagnoli e Schlafer (2006). Estes autores descrevem o desenvolvimento sexual como evento anterior e posterior ao nascimento, quanto aos órgãos genitais externos, portanto, este desenvolvimento encontrase completado aos 45 dias pós-coito.

A morfometria foi analisada e os resultados morfométricos tabulados na Tabela 1 .

O diâmetro biparietal dos fetos é em média de $3,7 \mathrm{~cm} \pm 0,6$ e o perímetro abdominal é em média de $8,0 \mathrm{~cm} \pm 1,0$. Estes achados, segundo alguns autores (CASTRO, et. al., 2011; TEXEIRA et. al., 2009) são dados valiosos frequentemente usados para calcular a idade gestacional dos fetos nos exames de ultrasonografia, auxiliando desse modo, o diagnóstico de diversas relacionadas esta faixa etária. 
Tabela 1. Morfometria de fetos de cães da raça Pinscher aos 45 pós-coito.

\begin{tabular}{lrrrrr}
\hline Medidas & Feto 01 & Feto 02 & Feto 03 & Feto 04 & \multicolumn{1}{c}{ Média } \\
\hline Peso(g) & 58,8 & 77,62 & 40,1 & 51,2 & $56,9 \pm 15,7$ \\
Altura(cm) & 3,10 & 3,01 & 3,38 & 3,05 & $3,1 \pm 0,9$ \\
Comprimento total (cm) & 17,5 & 18,8 & 17 & 18,2 & $18 \pm 0,9$ \\
Crown-rump CR (cm) & 10,2 & 11,1 & 8,7 & 10,0 & $10 \pm 0,9$ \\
Comprimento cefálico (cm) & 4,6 & 4,2 & 4,0 & 4,1 & $4,2 \pm 0,2$ \\
Comprimento da cauda (cm) & 3,5 & 4,0 & 3,4 & 2,9 & $3,4 \pm 0,4$ \\
Perímetro ocular (cm) & 0,7 & 0,7 & 0,5 & 0,4 & $0,5 \pm 0,1$ \\
Comprimento auricular(cm) & 1,1 & 0,8 & 0,5 & 1,0 & $0,8 \pm 0,2$ \\
Perímetro torácico (cm) & 10,0 & 11,2 & 8,4 & 9,0 & $9,6 \pm 1,2$ \\
Perímetro Abdominal (cm) & 9,2 & 10,3 & 8,0 & 8,2 & $8,9 \pm 1,0$ \\
Diâmetro Biparietal DBP(cm) & 3,8 & 4,6 & 3,0 & 3,6 & $3,7 \pm 0,6$ \\
Comprimento Céfalo-Caudal CCC (cm) & 11,4 & 11,1 & 7,0 & 8,2 & $9,4 \pm 2,1$ \\
\hline
\end{tabular}

Fonte: Elaboração dos autores.

O comprimento céfalo-caudal (CCC) é de $9,4 \pm 2,1 \mathrm{~cm}$ e a altura é, em média, de $3,1 \mathrm{~cm} \pm 0,2$. Segundo Texeira et al. (2009), a tomada de medida em exames ultrasonográficos do comprimento céfalo-caudal (CCC) é dificultada devido ao feto geralmente apresentar flexão lateral ou dorsoventral. O comprimento de ossos, que determinem a altura, como o úmero ou fêmur, também não apresentam nitidez no sonograma. Isso dificulta diagnóstico e tratamento de doenças quando se utiliza o ultrasom. Por esse motivo, os dados apresentados de CCC e altura são de grande valor para guiar o tratamento adequado de anomalias, que afetem estes órgãos.

Dados obtidos da Confederação Brasileira de Cinofilia (2007) demonstram que o comprimento da cabeça (medido da trufa ao occipital) corresponde à metade do comprimento da linha superior (medido da cernelha à raiz da cauda). Existe ainda uma relação entre a altura e o comprimento que fazem os fetos desta raça parecer o mais quadrado possível. Mesmo que, neste caso, os fetos observados apresentem uma relação entre o comprimento da cabeça e da linha superior, por não apresentarem diferença significante (Figura 1), não é adequado relacionar proporções em indivíduos em crescimento com proporções de indivíduos adultos. Segundo Peres Neto, Valentin e Fernandez (1995), durante o desenvolvimento dos organismos existem variações nas proporções que os permite manter-se funcionalmente ativo. Por este motivo, é um erro estabelecer uma mesma relação entre um indivíduo em desenvolvimento e um adulto.

Portanto, como nenhum dos fetos apresentou qualquer sinal de anormalidade congênita, os dados aqui apresentados podem ser usados como padrões de normalidade da raça Pinscher aos 45 dias póscoito, guiando exames clínicos de rotina que visem controlar a qualidade da gestação nesses animais. Estes dados são importantes parâmetros nas análises ultrasonográficas, por definirem o estado normal em que estes animais são encontrados in vivo. Por esse motivo, a padronização de valores que caracterizem a gestação da raça pinscher deve levar em conta estes dados morfométricos.

É importante notar ainda, que é incorreto o estabelecimento de relações proporcionais iguais entre indivíduos adultos e indivíduos em crescimento. 
Figura 1. Relação entre a metade do comprimento da linha superior e o comprimento cefálico dos fetos analisados. Os dados não apresentaram diferença significante. Foi realizado o teste Two-way ANOVA, com um nível de confiança $95 \%$.

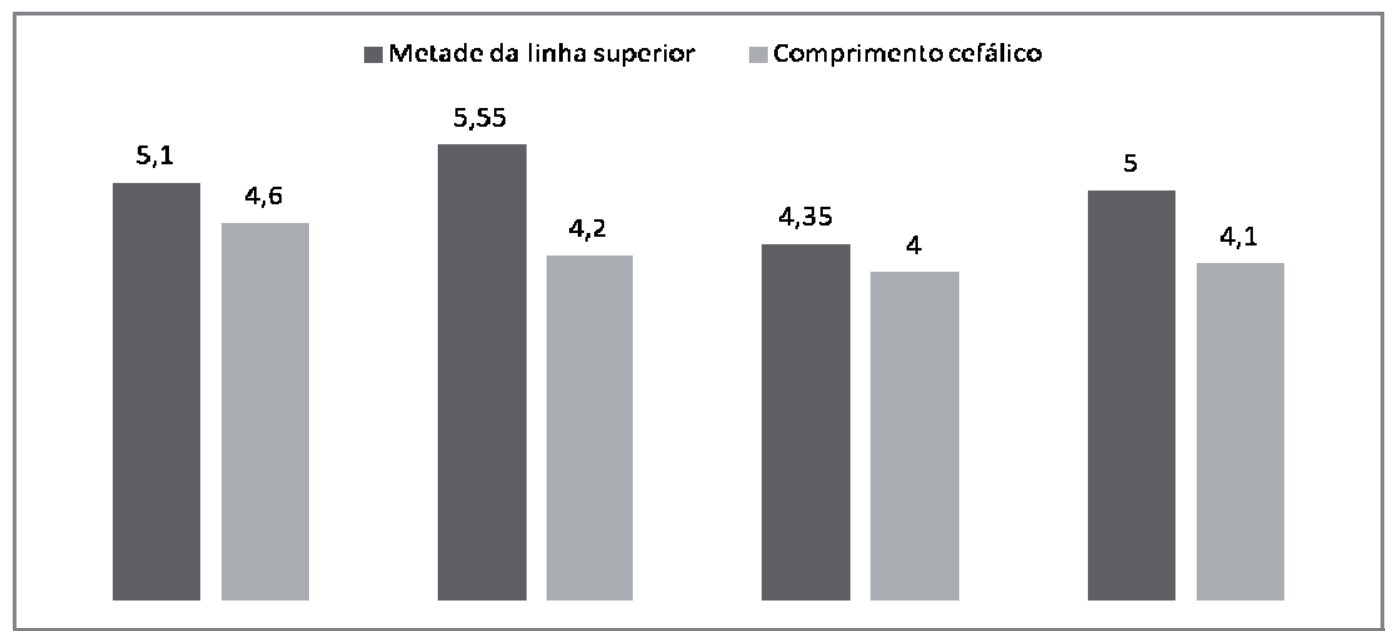

Fonte: Elaboração dos autores.

\section{Referências}

BARRETO, C. S. Avaliação de filhotes caninos. 2003. Tese (Doutorado em Medicina Veterinária, Reprodução Animal) - Faculdade de Medicina Veterinária e Zootecnia, Universidade de São Paulo, São Paulo.

BURBIDGE, H. M.; THOMPSON, K. C.; HODGE, H. Post natal development of canine caudal cervical vertebrae. Research in Veterinary Science, Oxford, v. 59, n. 1, p. 35-40, 1995.

CASTRO, V. M.; MAMPRIM, M. J.; LOPES, M. D.; SARTOR, R. Acompanhamento da gestação em cadelas pelo exame ultrassonográfico: revisão de literatura. Veterinária e Zootecnia, v. 18, n. 1, p. 9-18, 2011.

CONFEDERAÇÃO BRASILEIRA DE CINOFILIA. Padrão oficial da raça Pinscher: Zwergpinscher, 2012. Disponível em: <http://www.cbkc.org/padroes/pdf/ grupo2/pinscher.pdf $>$. Acesso em: 12 dez. 2012.

DYCE, K. M.; SACK, W. O.; WENSING, C. J. G. Tratado de anatomia veterinária. 2. ed. Rio de Janeiro: Guanabara Koogan, 1997.

EDMONDSON, S. British miniature pinscher. Lancashire: Mayfields, 2007. 126 p.

FERREIRA, R. R.; MACHADO, M. L. S.; AGUIAR, J.; SPANAMBERG, A.; BIANCHI, S. P.; OLIVEIRA, E. C.; DRIEMEIER, D. Displasias foliculares ligadas à cor da pelagem em cães: displasia folicular dos pêlos pretos e alopecia por diluição da cor. Act Sci Vet., Rio Grande do Sul, v. 35, n. 1, p. 119-124, 2007.
KERSWELL, K. J.; BENNETT, P.; BUTLER, K. L.; HEMSWORTH, P. H. The relationship of adult morphology and early social signaling of the domestic dog (Canis familiaris). Behav. Proc., Victoria, v. 81, p. 376-382, 2009.

KOLBER, M.; BORELLI, V. Dimensões dos rins de cães (Canis familiaris, Linneaus - 1758) da raça pastor alemão- ultrassonografia. Rev Inst Ciênc Saúde, São Paulo, v. 23, n. 1, p. 19-24, 2005.

LEITE, L. G. Acompanhamento gestacional em cadelas das raças yorkshire terrier e boxer por ultra-sonografia. 2003. Dissertação (Mestrado em Ciências Veterinárias) Faculdade de Veterinária do Ceará, Ceará.

MANDARIM DE LACERDA, de L. C. A. Manual de qualificação morfológica: morfometria, alometria, estereologia. Rio de Janeiro: CEBIO, 1994. 98 p.

MOURA, C. S.;GUERRA, M. M. P.; SILVA JÚNIOR, V. A.; SILVA, C. G. C.; CAJU, F. M. Histomorfometria do parênquima testicular de cães adultos tratados com ivermectina. Ciênc. Vet. Tróp., Recife, v. 9, n. 2-3, p. 8796, 2006.

PENNA, M. A. H. Avaliação das características morfológicas e morfométricas dos pêlos de roedores da mata atlântica do estado de São Paulo. 2009. Tese (Doutorado em Ciências Biológicas) -Universidade Estadual Paulista, São Paulo. 
PERES-NETO, P. R.; VALENTIN, J. L.; FERNANDEZ, F. A. S. (Ed.). Tópicos em tratamento de dados biológicos. Rio de Janeiro: UFRJ, 1995. p. 57-89. (Série Qecologia Brasiliensis, 2).

ROMAGNOLI, S.; SCHLAFER, D. H. Disorders of sexual differentiation in puppies and kittens: a diagnostic and clinical approach. Vet Clin Small Anim., Padua (Itália), v. 36, n. 3, p. 573-606, 2006.
STULBERG,D.; COOPERMAN,D.R.;WALLENSTEN, R. The natural history of legg-calvé-perthes disease. $J$ Bone Joint Surg Am., Ontario, v. 63, n. 7, p. 1095-1108, 1981.

TEIXEIRA, E. S. Princípios básicos para a criação de cães. São Paulo: Nobel, 2000.

TEXEIRA, M. J.; SOUZA, D. M. B.; MELO, K. C. M.; WISCHRAL, A. Estimativa da data de parto em cadelas Rottweiler através de biometria fetal realizada por ultrassonografia. Ciência Animal Brasileira, Goiânia, v. 10, n. 3, p. 853-861, 2009. 